\title{
IMPLICAÇÕES DA CONSCIÊNCIA FONOLÓGICA NA AQUISIÇÃO FONOLÓGICA DE L2 EM NÍVEL DE PERCEPÇÃO
}

\section{IMPLICATIONS OF PHONOLOGICAL AWARENESS IN L2 PHONOLOGICAL ACQUISITION AT PERCEPTION LEVEL}

\begin{abstract}
Almir Anacleto de Araújo Gomes ${ }^{7}$
RESUMO: Este trabalho procurou compreender e discutir como a consciência fonológica - CF - pode contribuir para a aquisição fonológica de segunda língua - L2. A discussão deu-se a partir de uma investigação teórica, tendo como suporte pesquisas empíricas sobre aquisição fonológica de L2, com foco na percepção ou compreensão, com o intuito de perceber as convergências e divergências entre tais investigações. Observou-se que, em nível de percepção, a CF atua de maneira positiva na aquisição fonológica de L2, contribuindo para uma comunicação mais efetiva, tendo em vista a atuação da CF na compreensibilidade e inteligibilidade dos sons da L2. Assim, os dados, resultados e discussões dos trabalhos observados confirmam-nos a importância da CF na aquisição fonológica de L2, bem como da sua relevância nas investigações no tocante à aquisição de formas linguísticas específicas de L2. Nesse sentido, a CF habilita os indivíduos a terem uma visão mais ampla do sistema fonológico de sua L1, do sistema fonológico da L2, assim como das semelhanças e diferenças entre esses sistemas fonológicos. Dotados dessa habilidade, os aprendizes têm um desempenho mais eficiente na compreensão da L2.
\end{abstract}

PALAVRAS-CHAVE: Consciência Fonológica. Aquisição Fonológica. Aquisição de L2. Percepção

\begin{abstract}
This work seeks to understand and discuss how phonological awareness can contribute to L2 phonological acquisition. The discussion is based on a theoretical investigation, supported by several empirical research on L2 phonological acquisition which focused on L2 perception or comprehension. Its purpose is to understand the convergences and divergences among such investigations and thus build an insight of how phonological awareness can contribute to L2 phonological acquisition. It was detected that at the level of perception, phonological awareness acts positively in the L2 phonological acquisition, contributing to a more effective communication, in view of the phonological awareness performance in the L2 sounds comprehensibility and intelligibility. Therefore, data, results and discussions of the analysed papers confirm the importance of phonological awareness in L2 phonological acquisition, as well as its relevance regarding the acquisition of L2-specific linguistic forms. In this sense, phonological awareness enables learners to have a broader view of the L1 phonological system, the L2 phonological system, as well as the similarities and differences between them. Having this ability, learners perform more efficiently on L2 comprehension.
\end{abstract}

KEYWORDS: Phonological Awareness. Phonological Acquisition. L2 Acquisition. Perception

\section{Introdução}

Este trabalho buscar compreender e discutir o papel da Consciência Fonológica (CF) no processo de aquisição fonológica de segunda língua (L2), em termos de compreensão ou percepção, a partir de dados, resultados e discussões de pesquisas em aquisição fonológica de L2 que analisam a CF como uma variável do processo de aquisição. Outrossim, procuraremos analisar como esse processo de aquisição fonológica de L2 pode ser compreendido ou explicado a partir da $\mathrm{CF}$, numa tentativa de verificar a importância de se considerar a $\mathrm{CF}$ como uma variável do processo de aquisição fonológica de uma L2.

Para a compreensão do papel da CF na aquisição fonológica de L2, nós buscamos fazer um levantamento de trabalhos acadêmicos sobre a aquisição fonológica de L2, tendo como critério básico ser um trabalho de pesquisa em aquisição de algum aspecto fonológico de L2 e que tenha considerado a contribuição da CF no processo de aquisição, mesmo que se tenha utilizado no trabalho outra nomenclatura equivalente ao que está se denominando de $\mathrm{CF}$ neste trabalho. É importante reiterar que o termo CF está sendo utilizado neste trabalho como

\footnotetext{
${ }^{7}$ Doutor e mestre em Linguística pela Universidade Federal da Paraíba (UFPB). Professor da Universidade Federal de Campina Grande (UFCG). E-mail: almir.ufcg@gmail.com
} 
um termo guarda-chuva que abarca outros termos utilizados nas pesquisas selecionadas, entretanto com o mesmo sentido de conhecimento metafonológico.

Para a busca ${ }^{8}$ das investigações utilizamos o banco de teses e dissertações da Capes, a Biblioteca Digital Brasileira de Teses e Dissertações, o Portal de Periódicos da Capes, a base de dados eletrônicos da Universidade Estadual da Pensilvânia, o repositório de dissertações e teses da Brigham Young University, bem como a biblioteca da Concordia University de Montreal no Canadá.

Ao discutir a relação entre a CF e a percepção e compreensão dos sons da língua alvo na interfonologia do aprendiz de L2, estamos levando em conta a comunicação na língua alvo, ou seja, vislumbramos uma compreensão auditiva desses aprendizes que proporcione uma comunicação efetiva, sem grandes ruídos, isto é, que haja compreensibilidade entre os interlocutores.

Observamos que aprendizes de L2 que são mais conscientes de suas próprias produções na L2 e que possuem maior consciência sobre a fonologia da L2 como um todo percebem e produzem os sons da L2 com mais precisão (BAKER e TROFIMOVICH, 2006; NAVEHEBRAHIM, 2012; KIVISTÖ-DE SOUZA, 2015; 2017). Sendo assim, uma percepção do sistema fonológico da L2 é também um propulsor de uma produção mais adequada dos sons da L2.

Nesse sentido, a CF proporciona uma melhor compreensão dos sons da L2, através da eliminação ou redução das interferências do sistema fonológico da L1 do aprendiz. Essa habilidade de notar as divergências entre os sistemas fonológicos permite uma dependência cada vez menor da L1 para a compreensão dos sons na L2 e um movimento gradual de independência na recategorização dos sons conforme o sistema fonológico da L2, na interfonologia dos aprendizes de L2 e proporciona uma melhor comunicação na interação oral na língua.

Dentro dessa perspectiva, compreendemos que a consciência da percepção dos sons e da sua relação com os demais sons no sistema fonológico da língua tem uma importância na aquisição e, consequentemente, na comunicação na língua alvo. Sendo assim, se levarmos em consideração falantes nativos de PB em processo de aquisição fonológica de inglês como L2, é possível que esses indivíduos possam conceber, a princípio, o som inicial da palavra thanks como /t/, /f/ ou /s/ devido a uma categorização fonética inadequada, dentro dos padrões sonoros da língua portuguesa (SAMUEL e KRALJIC, 2009).

Assim, os aprendizes ao perceberem os sons que estão a produzir comparam-nos com os sons da língua alvo e, a partir de uma tomada de consciência das divergências e convergências dos sistemas fonológicos das duas línguas. Com isso, tais falantes nativos de PB estariam aptos a recategorizar o som inicial da palavra mencionada como o $/ \theta /$, dentro dos padrões sonoros da língua alvo, por estarem conscientes do padrão fonológico da L2.

\section{Trabalhos que confirmam o papel positivo da CF na aquisição fonológica de L2 em termos de percepção}

Podemos confirmar essa concepção nos resultados de Navehebrahim (2012) sobre desvios da norma padrão da pronúncia de aprendizes de inglês como L2, nativos de língua persa no Irã. Os seus dados revelaram que um dos fatores que levam os iranianos a se desviarem da norma padrão da pronúncia da L2 é a incapacidade de perceber as diferenças entre os sistemas da língua inglesa e da língua persa. Além disso, os iranianos nativos de

\footnotetext{
${ }^{8}$ Buscamos os trabalhos a partir das seguintes palavras-chave: aquisição fonológica, instrução explícita, instrução formal, consciência fonológica, conhecimento metalinguístico, phonological acquisition, explicit instruction formal instruction, phonological awareness, metalinguistic knowledge, adquisición fonológica, instrucción explícita, instrucción formal, conciencia fonológica e conocimiento metalingüístico.
} 
língua persa, aprendizes de inglês como L2, parecem não ser capazes de ouvir e identificar todos os sons das palavras na língua alvo. O papel positivo da identificação das diferenças entre os sons que são da L1 dos aprendizes ou não e então, o desenvolvimento da percepção seletiva dos sons da L2 de forma a que se consiga ouvi-los de maneira adequada é confirmado por Kissling (2014).

Corroborando essa relação entre a percepção consciente dos sons e a sua produção, estudos mostram que a pronúncia de aprendizes de L2 é influenciada pela maneira como tais sons são percebidos, conforme podemos observar em Peleias (2009), que há uma relação entre a percepção e a produção da pronúncia da fricativa interdental surda por falantes nativos de $\mathrm{PB}$, aprendizes de língua inglesa como L2. Quando mencionamos que os aprendizes de L2 precisam perceber ou notar as diferenças entre os inventários fonológicos das duas línguas, a sua L1 e a língua em processo de aprendizagem, estamos nos referindo ao fato de que nem sempre essa percepção dos sons acontece de maneira adequada. Assim, aprendiz de uma L2, durante esse processo de interfonologia ou de aquisição da pronúncia da L2, pode perceber os sons da língua como semelhantes aos sons da sua L1, mesmo que eles não sejam.

Diante disso, os indivíduos que conseguem firmar os novos sons da L2 em uma nova categoria diferente dos sons da L1 são mais bem-sucedidos do que aqueles indivíduos que percebem os sons da L2 a partir de uma categoria sonora de sua L1. Portanto, percebemos a importância de reconhecermos as distinções sonoras da língua para uma melhor percepção e produção do seu inventário fonológico, bem como da CF para compreendermos o processo de aquisição fonológica de L2.

O referido trabalho investigou a relação entre a produção e a percepção da consoante fricativa interdental surda $[\theta]$ em um grupo de três falantes nativos de PB, aprendizes de inglês como L2. Os resultados dessa investigação, com base em instrumentais da análise fonético-acústica, mostraram que 53,3\% da amostra de percepção dos brasileiros tenderam a perceber o som [ $\theta$ ] como o alvo e $40 \%$ da amostra perceberam como sendo o som da consoante oclusiva alveolar desvozeada [t].

Uma análise de percepção do referido som também foi realizada com os mesmos sujeitos, e por fim, uma avaliação, com 31 'juízes' falantes nativos de inglês norte-americano, da produção do som em estudo. Para efeito de análise, foi realizado um cruzamento desses dados com o intuito de verificar se há a relação entre a percepção e a produção desse som. A autora apresenta a questão da percepção e da produção de fala, modelo de aprendizagem da fala, o papel do fator idade, a interferência da fala no aprendizado de fonemas de L2, a descrição de diversos fonemas na língua inglesa e na língua portuguesa, apresentando inclusive uma análise fonético-acústica (PELEIAS, 2009).

Salientamos que, embora Peleias (2009) leve em consideração a comparação da produção dos aprendizes de inglês como L2 com a fala de um nativo de uma variedade da língua inglesa, a nossa discussão aqui não leva em conta essa proximidade de produção sonora. Quando discutimos a CF como um possível favorecedor da aquisição fonológica de L2, acreditamos na aquisição de uma pronúncia que não restrinja a comunicação efetiva entre falantes não nativos ou entre falantes nativos e não nativos da língua alvo.

Nessa mesma perspectiva, Sandes (2010) apresenta a dificuldade de falantes nativos de PB, aprendizes de espanhol como L2, alunos do curso de Letras com habilitação em língua espanhola de uma universidade de São Paulo, em perceber as diferenças entre os sistemas fonológicos de ambas as línguas. Essa inabilidade em perceber as sutis diferenças entre os inventários fonológicos do PB e da língua espanhola pode levar a uma produção sonora não esperada e inadequada na língua alvo.

Essa dificuldade com o sistema fonológico da L2 pode ser compreendida com base nos conceitos de 'surdez fonológica' de Polivanov ([1931] 1978) e o de 'crivo fonológico', de Trubetzkoy (1939), segundo os quais, os aprendizes podem ser fonologicamente surdos e 
utilizar-se do crivo fonológico da L1 na produção de sons em L2, ou seja, um conceito complementa o outro. Nesse sentido, Sandes (2010) corrobora que o sistema fonológico da língua materna é apresentado de forma a atuar como um "crivo fonológico" ou filtro de percepção pelo qual as informações sonoras da L2 passam, de modo que o aprendiz de L2 interpreta tais sons de maneira equivocada, conforme o sistema sonoro de sua L1.

O corpus de Sandes (2010) é formado por 76 palavras em língua espanhola contendo os sons oclusivos sonoros [b, d, g] e seus alofones [ $\beta, \gamma, \mathrm{\gamma}, \mathrm{\gamma}$, como em barba ['bar $\beta \mathrm{a}$ ], delgado [del'yaðo] e rasgado [ras 'yaðo] e os sons nasais [m, n, n], como em mango ['mãngo], comen ['komẽn] e pan ['pãn], em vários contextos controlados.

Então, sendo a nasalidade uma característica do PB, por meio do crivo fonológico, nativos de $\mathrm{PB}$, aprendizes de espanhol como $\mathrm{L} 2$ podem não perceber as características acústico-articulatórias da L2 como são de fato (SANDES, 2010). A compreensão desse sistema sonoro se dá sob o filtro perceptivo da L1, pois a percepção dos sons e suas distinções na L2 podem ser significativamente influenciadas pela L1 do indivíduo (GUIMARÃES, 2012). Compreendemos, assim, que a proximidade entre a L1 e a L2 pode ter levado esses informantes a não categorizarem os sons conforme a L2, mas como um som da L1.

Os resultados de Sandes (2010) demonstram que o desenvolvimento da CF apresenta um papel positivo na aquisição fonológica da L2, permitindo que os aprendizes possam perceber os sons da L2 sem apoiar-se no inventário fonológico da L1. Percebemos, portanto, a importância do desenvolvimento da CF no aprendiz de L2 para que possam desenvolver a habilidade de reconhecer e reconfigurar os sons da interfonologia da L2 conforme as categorias do inventário fonológico da língua alvo.

Dentro dessa concepção, o trabalho de Pagoto de Sousa (2012) analisou a produção e a percepção de cinco alunas do quarto ano do curso de Licenciatura em Letras (Inglês/Português), falantes nativas de PB, dos sons vocálicos: /i/ e /I/; /E/ e /æ/; /ə/; /a/ e /o/; $/ \widetilde{\sigma} / \mathrm{e} / \mathrm{u} / \mathrm{e}$ da consoante fricativa dental surda $/ \theta /$ e da fricativa dental sonora $/ \mathrm{\delta} /$, que supostamente podem ser substituídas pela consoante fricativa alveolar desvozeada /s/ e pela fricativa labiodental desvozeada /f/ ou pela consoante oclusiva alveolar desvozeada / $t$ / e pela oclusiva alveolar vozeada /d/, por não serem fonemas do $\mathrm{PB}$ e por apresentarem características próximas a fonemas do $\mathrm{PB}$.

Os resultados da pesquisa revelaram que houve alguns problemas gerais na identificação e diferenciação dos sons, principalmente em relação às vogais altas anteriores $/ \mathrm{i} / \mathrm{e} / \mathrm{I} / \mathrm{h} / \mathrm{u} / \mathrm{e} / \widetilde{J} / \mathrm{e}$ poucos erros em relação à identificação e diferenciação das consoantes fricativas $/ \theta / \mathrm{e} / \mathrm{\delta} /$, mas que, no geral, as informantes não apresentaram grandes problemas no teste de percepção (PAGOTO DE SOUSA, 2012). A autora justifica esses problemas no fato de os dados gravados para os testes de percepção tenham sido feitos por um falante nativo de PB.

Compreendemos, por outro lado, que o input para a coleta de dados da pesquisa, tendo base na fala de um nativo de PB, é mais realístico para a maioria dos aprendizes brasileiros de L2, considerando que dificilmente encontramos professores falantes nativos da L2 no contexto brasileiro. Salientamos que, para a nossa coleta de dados, optamos por oferecer um input gravado por falantes nativos de $\mathrm{PB}$, conforme detalharemos no capítulo 03, mais adiante.

Dentre os trabalhos que pesquisaram o papel da CF na percepção e aquisição fonológica de L2, podemos citar também Silva (2014b), que investigou os processos de percepção e produção das vogais médias /e/ - /E/ e /o/ - /o/ em sílaba tônica e a elevação das vogais médias /e/ e /o/ em sílaba pretônica do PB por imigrantes adultos falantes nativos de língua espanhola da América Latina e residentes em Porto Alegre - RS, com idade entre 18 e 59 anos e que tenham migrado para o Brasil a partir dos 18 anos de idade, aprendizes de português como L2. 
Quanto à variável instrução formal, dois fatores foram considerados: o informante possuir contato com um português formal por ter frequentado ou estar frequentando algum curso de idiomas ou cursos de ensino fundamental, médio, graduação ou de pós-graduação no Brasil ou o informante não ter tido contato formal com o português. A hipótese levantada foi a de que o desempenho dos falantes não nativos que tiveram acesso ao português formal seria superior aos que não tiveram tal acesso, principalmente nas atividades envolvendo o contraste fonológico entre vogais médias tônicas.

Os dados de Silva (2014b) mostraram que os informantes que tiveram acesso à instrução formal do português como L2, aparentemente, apresentam um desempenho superior na percepção do contraste entre vogais médias tônicas. Tais dados confirmam a hipótese de que a instrução formal atua de maneira significativa na percepção fonológica, levando nativos de espanhol, aprendizes de português como L2, a apresentarem um melhor desempenho para discriminar e identificar o contraste entre vogais médias tônicas. Portanto, a instrução formal da fonologia da L2 pode desenvolver a CF na língua alvo e levar os aprendizes a apresentar uma pronúncia mais acurada.

O estudo de Wong, Mok, Chung, Leung, Bishop e Chow (2017), que examinou a relação entre várias habilidades fonológicas subjacentes à percepção de formas reduzidas do inglês, como no caso de contração, elisão, assimilação etc., com alunos de graduação chineses, aprendizes de inglês como L2, confirmou que a capacidade de perceber as formas fonologicamente reduzidas foi um indicador significativo da compreensão da fala conectada do falante nativo de língua inglesa.

Os dados mostraram que a capacidade dos aprendizes de formar representações fonológicas é crucial para armazenar formas reduzidas no léxico mental e aprender regras fonológicas abstratas. Dessa forma, indivíduos com boa consciência fonêmica têm representações fonológicas mais detalhadas, o que pode ser útil para a recuperação de fonemas que foram reduzidos na fala corrida. Assim, os autores corroboram a ideia de que a discriminação melhorada dos contrastes fonológicos em palavras isoladas pode promover a conscientização das unidades fonológicas no nível metalinguístico.

O reconhecimento de vocabulário e o reconhecimento de partes de palavras foram indícios significativos de percepção de formas reduzidas de inglês nativo. Portanto, percebemos que a $\mathrm{CF}$, nesse caso, no nível da consciência fonêmica, contribuiu para uma compreensão mais acurada da fala corrida e interconectada do falante nativo de língua inglesa, por aprendizes de inglês como L2, falantes nativos de chinês.

O papel da CF também foi investigado por Kurt, Medlin e Tessarolo (2014), em cujo trabalho foram verificados os efeitos da instrução explícita na percepção de padrões de entonação ambígua, quanto à prosódica por aprendizes de inglês como L2, falantes nativos de mandarim, japonês, espanhol e árabe.

Os dados, no geral, mostram que, entre o pré e o pós-teste, o aumento nos escores foi maior para o grupo experimental $(7,29 \%)$ do que para o grupo controle $(4,55 \%)$. Ou seja, para o grupo que recebeu instruções explícitas durante a fase de treinamento (em torno de 5 a 6 horas, distribuídas ao longo de 4 semanas) a melhora da percepção foi superior à do grupo que não recebeu a instrução explícita sobre o fenômeno investigado. Embora os resultados não tenham sido considerados estatisticamente significativos ( $\mathrm{p}>0,05)$, os cálculos baseados em porcentagem corroboram com o fato de que o ensino explícito de padrões de entonação para aprendizes com pouca proficiência pode ser útil na compreensão da prosódia da língua alvo.

Corroborando esse raciocínio teórico, Gómez Lacabex e Gallardo del Puerto (2014) investigaram o efeito do treinamento fonético formal (treinamento baseado na percepção, treinamento baseado na produção e exposição ao sotaque nativo) na percepção do som schwa lexical do inglês de 75 alunos do sexto ano, falantes nativos de espanhol e basco e aprendizes de inglês como L2, distribuídos em três grupos de 25 indivíduos cada. 
Segundo os autores, dois grupos receberam treinamento fonético diferente: o primeiro grupo recebeu treinamento com base em tarefas de percepção, incluindo tarefas de discriminação e identificação, enquanto que o segundo grupo recebeu treinamento com base em prática de ouvir e repetir. Os informantes ouviam a palavra ao mesmo tempo em que viam a palavra escrita na tela e em seguida repetiam. A fala dos informantes era gravada e os estes deveriam ouvir a sua fala em seguida, ao menos uma vez ou quantas vezes mais desejassem. O terceiro grupo não recebeu treinamento fonético, mas recebeu aulas de inglês, artes e artesanato com um professor falante nativo de língua inglesa.

Ainda de acordo com Gómez Lacabex e Gallardo del Puerto (2014), o professor monitorava as produções ao longo das sessões e fornecia feedback positivo aos informantes que tentavam produzir uma vogal reduzida com sucesso, além de lembrar aos alunos que não conseguiam produzir a vogal reduzida, as dicas de pronúncia apresentadas na sessão introdutória.

Os resultados atestaram os efeitos positivos do treinamento sobre a consciência perceptiva dos aprendizes a respeito da ocorrência de $s c h w a$ em sílabas não tônicas em inglês. Além disso, os grupos que passaram por intervenção fonética explícita exibiram uma consciência dos sons investigados de forma mais significativa do que o grupo com exposição nativa, que permaneceu em níveis de desempenho casual nos contextos nos quais os outros grupos apresentaram melhora, como no caso das vogais plenas (GÓMEZ LACABEX e GALLARDO DEL PUERTO, 2014).

Os autores afirmam que os resultados atestam que os contextos de aprendizagem em sala de aula podem se beneficiar dos protocolos de treinamento fonético explícitos, como discriminação, identificação ou imitação, auxiliados por computador. Além disso, esse estudo fornece evidências de que os dois tipos diferentes de treinamento fonético utilizados podem aumentar a consciência da percepção dos alunos sobre a ocorrência de som L2. Portanto, acreditamos que tais resultados são importantes para compreendermos a relação complexa entre a percepção da fala e a sua produção dentro do domínio de treinamento explícito.

O papel da consciência como atenção também foi investigado Darcy, Mora e Daidone (2014) que buscaram verificar até que ponto o controle de atenção e a inibição 9 estariam associados ao processamento fonológico da L2 na percepção e na produção. Segundo a compreensão das autoras, a capacidade de mudar a atenção entre diferentes dimensões relevantes para uma determinada tarefa desempenha um papel importante no aprendizado de L2. Já a inibição é responsável por impedir o apoio na L1 ao comunicar-se na L2, minimizando efetivamente a interferência fonológica da L1 na percepção e produção da fala na L2.

Os resultados da pesquisa realizada com 16 indivíduos falantes nativos de espanhol e aprendizes de inglês como L2 e 18 falantes nativos de inglês e aprendizes de espanhol como L2, sugerem que os aprendizes de L2 com maior capacidade de controle de atenção foram mais hábeis em perceber e usar as características contrastivas das vogais e das consoantes investigadas (Quadro 03), na categorização dos estímulos de fala da L2.

\begin{tabular}{|c|c|c|c|c|c|}
\hline & Test consonant & Control consonant & test vowel & Control vowel & Common contrasts \\
\hline $\begin{array}{l}\text { L1-Spanish, } \\
\text { L2-English }\end{array}$ & $/ \mathrm{J} /$ vs. $/ \mathrm{t} \mathrm{d} /$ & /d/ vs. /f/ & /i/ vs. /I/ & /e/ vs. /eI/ & $\begin{array}{l}\text { /a/ vs. /i/, } \\
/ \mathrm{t} / \text { vs. /d/ }\end{array}$ \\
\hline $\begin{array}{l}\text { L1-English, } \\
\text { L2-Spanish }\end{array}$ & $/ \mathrm{d} / \mathrm{vs} . / \mathrm{f} /$ & $/ \mathrm{J} /$ vs. $/ \mathrm{t} \mathrm{g} /$ & /e/ vs. /eI/ & /i/ vs. /I/ & $\begin{array}{l}\text { /a/ vs. /i/, } \\
\text { /t/ vs. /d/ }\end{array}$ \\
\hline
\end{tabular}

FONTE: Darcy, Mora e Daidone (2014, p. 119).

\footnotetext{
${ }^{9}$ Tradução livre
} 
Assim, os resultados de Darcy, Mora e Daidone (2014) sugerem que o controle de atenção, além de desenvolver representações perceptivas mais precisas para os sons da L2, permite que os aprendizes possam trazer informações relevantes para o primeiro plano, durante o processamento da fala, mantendo informações irrelevantes em segundo plano, sendo potencialmente importante para o sucesso da aprendizagem fonológica de L2.

O trabalho de Gordon, Darcy e Ewert (2013) também investigou os benefícios da instrução explícita, como um agente do direcionamento da atenção dos aprendizes e conscientização das características segmentais e suprassegmentais da L2, na aquisição de pronúncia em L2. Os seus resultados apontaram que a instrução fonética explícita beneficia os aprendizes de L2 em geral; mesmo a instrução tendo sido apenas de um componente de pronúncia, relativamente limitado no tempo, em uma sala de aula principalmente comunicativa, pode levar a resultados benéficos na produção dos alunos.

O trabalho de Silveira (2004) que investigou quão eficiente seria o ensino da pronúncia na aquisição de consoantes do inglês /p/, /b/, /t/, /d/, /k/, /g/,/f/, /v/, /dz/, /m/, /n/,/y/ em posição de final de palavra por alunos brasileiros, em nível iniciante de proficiência na língua inglesa, tanto em nível de percepção quanto em nível de produção, também demonstrou que a instrução formal contribuiu de maneira positiva na percepção das consoantes em posição final de palavras.

Com o objetivo de avaliar a inteligibilidade da língua inglesa como língua franca, por aprendizes de inglês como L2, falantes nativos de PB, Becker e Kluge (2014) conduziram um estudo com 80 estudantes do curso de graduação em Letras inglês - português de uma universidade pública do sul do Brasil. Os informantes da pesquisa haviam recebido, ao menos, 450 horas de aulas de língua inglesa e para a pesquisa receberam estímulo oral de 8 falantes de inglês de diferentes contextos de L1 (estadunidenses, chineses falantes nativos de mandarim, alemães e japoneses).

Os resultados apontaram que a produção de sons vocálicos/consonantais individuais foi, de fato, apontada pelos ouvintes brasileiros como o principal fator de ininteligibilidade para a fala de alemães, chineses e japoneses. Para os estadunidenses, a taxa de fala foi apontada como o fator de maior causa da ininteligibilidade, enquanto que o ritmo foi considerado o segundo fator, como podemos observar na Tabela 01, a seguir:

Tabela 01: Relação entre instrução formal e inteligibilidade por aprendizes de inglês como L2, falantes nativos

\begin{tabular}{ccccccc}
\multicolumn{7}{c}{ de PB, segundo os dados de Becker e Kluge (2014) } \\
\hline Pairs & $\begin{array}{c}\text { Numbers of } \\
\text { listeners }\end{array}$ & $\begin{array}{c}\text { Minimum } \\
\text { number of } \\
\text { correct words }\end{array}$ & $\begin{array}{c}\text { Maximum } \\
\text { number of } \\
\text { correct words }\end{array}$ & $\begin{array}{c}\text { Average } \\
\text { number of } \\
\text { correct words }\end{array}$ & Intelligibility & $\begin{array}{c}\text { Standard } \\
\text { Deviation }\end{array}$ \\
\hline Germans & 20 & 37 & 66 & 53.3 & 77.2 & 8.773 \\
Americans & 20 & 37 & 66 & 53.1 & 77.0 & 9.706 \\
Chinese & 20 & 35 & 64 & 55.3 & 80.1 & 7.328 \\
Japanese & 20 & 31 & 58 & 42.3 & 61.3 & 7.637 \\
All & 80 & 31 & 66 & 51.0 & 73.9 & 9.716 \\
FONTE: Becker e Kluge $(2014$, p. 55). & & & &
\end{tabular}

Os dados nos revelam que, por se tratar de um grupo de informantes que está em preparação para se tornarem professores de língua e que, portanto, receberam instrução formal da língua alvo, são indivíduos com provável CF mais desenvolvida do que um aprendiz comum de L2, o que pode ter influenciado na inteligibilidade dos falantes de diferentes línguas maternas. Além disso, percebemos a importância do desenvolvimento da CF por aprendizes de L2 e, principalmente, por aqueles que estão em formação para serem professores da língua alvo.

Dentro dessa perspectiva, Silveira e Alves (2009) investigaram o papel da instrução explícita na aquisição do morfema -ed do passado simples ou particípio passado dos verbos 
regulares da língua inglesa, por aprendizes brasileiros de inglês como L2, a partir de dois experimentos: no primeiro experimento verificaram o papel da instrução explícita na produção de verbos com a marca -ed, enquanto que no segundo experimento, analisaram tanto a produção quanto a percepção do morfema -ed.

Os resultados de produção do primeiro experimento mostraram-se positivos para a instrução formal tanto no primeiro pós-teste quanto no segundo, confirmando inclusive um efeito de longa duração da instrução explícita na produção do fenômeno investigado, conforme podemos observar na Tabela a seguir:

Tabela 02: Resultados do pré-teste e pós-teste para as pronúncias [t] e [d] do primeiro experimento de Silveira e

\begin{tabular}{|ccccccc|}
\hline & Pré-Instrução & \multicolumn{3}{c|}{ Pós-Instrução 1 } & Pós-Instrução 2 \\
\cline { 2 - 8 } & Entrevista & Leituras & $\frac{\text { Entrevista }}{16,7}$ & $\frac{\text { Leituras }}{50,9}$ & Entrevista & Leituras \\
Formas-alvo & 24 & 16,9 & 41,3 & 61,1 \\
Diferentes do alvo & 76 & 83,3 & 49,1 & 43,1 & 48,7 & 38,9 \\
\hline
\end{tabular}

FONTE: Silveira e Alves (2009, p. 13).

Embora os resultados do segundo experimento demonstrem que a instrução explícita tenha sido mais favorável para a percepção (ganho de 35 pontos percentuais) do que para a produção (ganho de 14 pontos percentuais), esses dados ainda mostram que a instrução explícita se mostrou favorável tanto para a percepção quanto para a produção.

Os autores observam que o processo de aquisição do sistema fonológico de uma L2 é complexo e que exige que o aprendiz tenha um determinado grau de consciência dos sons da L2, bem como das suas diferenças dos sons produzidos em sua L1. Nesse sentido, os resultados mostram que a instrução explícita pode contribuir para chamar a atenção dos aprendizes a respeito das diferenças entre os sistemas fonológicos da L1 e da L2, de maneira que tais aprendizes possam perceber de modo seletivo as formas-alvo do input na L2. A instrução explícita, então, desenvolveu a capacidade de noticing nos indivíduos.

$\mathrm{O}$ conceito de notar (noticing), pois, se torna central no processo de aquisição dos sons da L2, uma vez que é pré-requisito para que o aprendiz tenha consciência das diferenças entre os dois sistemas fonológicos, o que, segundo Silveira e Alves (2009), é uma premissa para a aquisição fonético-fonológica da L2. Nesse sentido, notar vai além de apenas perceber o sinal acústico. Notar é, acima de tudo, processar as diferenças entre os padrões do sistema fonético-fonológico da L1 e da L2, através de uma atenção seletiva.

O estudo de Van Dommelen e Husby (2008) teve como objetivo investigar a percepção de certos tons noruegueses ${ }^{10}$ por falantes nativos de mandarim que é uma língua tonal; e falantes de alemão, que não é uma língua tonal. O resultado demonstrou, sem novidades, que os falantes da língua tonal - mandarim - obtiveram um desempenho global melhor do que o dos falantes de alemão.

Além disso, os falantes nativos de alemão, do grupo experimental, também tiveram melhores resultados no teste após as aulas sobre a pronúncia e os tons do norueguês, revelando que os melhores resultados nos testes de discriminação e identificação dos tons da língua norueguesa alcançados pelos falantes nativos de alemão deram-se pelo desenvolvimento da CF nesse aspecto do norueguês.

O trabalho de Rocca (2003) investiga o desenvolvimento da percepção e produção de contornos entonacionais do inglês por 6 falantes nativos de PB, alunos de uma disciplina intitulada "Fonética e Fonologia: Entoação do Inglês" do curso de Letras - Inglês da PUC/SP, com base na conscientização das características do fenômeno investigado por meio da análise, manipulação e visualização do sinal sonoro, através de instrumentos de análise acústica da

\footnotetext{
${ }^{10} \mathrm{De}$ acordo com os autores, diferentemente de quase todas as outras línguas europeias, o norueguês utiliza tons lexicais no seu sistema fonológico.
} 
fala, e mais dois alunos (grupo controle), que cursaram a mesma disciplina, porém sem o recurso da tecnologia de fala.

Os resultados foram positivos para a melhoria na percepção e produção do fenômeno estudado, com destaque para o fato de que o uso de recursos complementares ao ensino de L2 tem muito a contribuir para um desenvolvimento mais eficiente da CF dos aprendizes de L2.

Embora o programa computacional utilizado por Rocca (2003) não tenha as mesmas finalidades que os programas de voz sintética que apresentaremos no capítulo 03 deste trabalho, compreendemos, assim como a autora citada, que os recursos tecnológicos nos quais os aprendizes possam manipular o input de maneira consciente têm muito a contribuir para o aprendizado da L2.

Por fim, percebemos o papel positivo da instrução explícita também nos resultados do trabalho de Huthaily (2008) que investigou os efeitos da instrução explícita na produção e percepção de consoantes do árabe em falantes nativos de inglês, aprendizes de árabe como L2. Podemos observar, no quadro a seguir, um resumo dos trabalhos que investigaram, com resultados positivos, o papel da CF na percepção do sistema fonológico da L2:

Quadro 02: Síntese dos trabalhos que investigaram o papel da CF na percepção sonora em L2, selecionados para

\begin{tabular}{|lcc|}
\multicolumn{4}{c}{ Pesquisa } & L1 & L2 \\
\hline Rocca (2003) & PB & Inglês \\
\hline Silveira (2004) & PB & Inglês \\
\hline Huthaily (2008) & Inglês & Árabe \\
\hline Van Dommelen e Husby (2008) & Mandarim/Alemão & Norueguês \\
\hline Peleias (2009) & PB & Inglês \\
\hline Silveira e Alves (2009) & PB & Inglês \\
\hline Sandes (2010) & PB & Espanhol \\
\hline Pagoto de Sousa (2012) & PB & Inglês \\
\hline Gordon, Darcy e Ewert (2013) & Não informada & Inglês \\
\hline Darcy, Mora e Daidone (2014) & $\begin{array}{c}\text { Espanhol } \\
\text { Inglês }\end{array}$ & Inglês \\
\hline Gómez Lacabex e Gallardo del Puerto (2014) & Basco e espanhol & Inglês \\
\hline & $\begin{array}{c}\text { Mandarim, japonês, } \\
\text { espanhol, árabe, } \\
\text { chinês e Coreano. }\end{array}$ & Inglês \\
Kurt, Medlin e Tessarolo (2014) & Francês & Espanhol \\
\hline Schwab e Llisterri (2014) & Espanhol & PB \\
\hline Silva (2014b) & Chinês & Inglês \\
\hline $\begin{array}{l}\text { Wong, Mok, Chung, Leung, Bishop e Chow } \\
\text { (2017) }\end{array}$ & & \\
\hline
\end{tabular}

Fonte: Dados da pesquisa (2018).

Em outros trabalhos (ALIAGA-GARCÍA e MORA, 2008; RUHMKE-RAMOS, 2009; PEROZZO, 2013), embora os dados apontem para um papel positivo da CF, não foi possível comprovar com dados estatísticos, conforme poderemos observar na próxima seção.

\section{Trabalhos que confirmam em parte o papel positivo da CF na aquisição fonológica de L2 em termos de percepção}

O trabalho de Aliaga-García e Mora (2008) investigou o papel da instrução explícita a respeito de quatro pares de sons $(/ \mathrm{p} /-/ \mathrm{b} /, / \mathrm{t} /-/ \mathrm{d} /$ em posição inicial de palavra + as vogais $/ \mathrm{i}: /-$

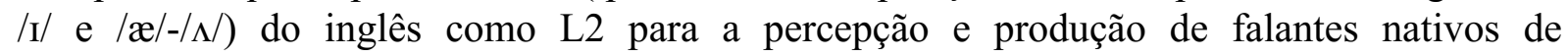


catalão/espanhol em contexto formal de aprendizagem na Universitat de Barcelona em Catalunha, Espanha. A escolha desses pares sonoros deu-se devido à maior dificuldade em sua percepção e produção por parte dos falantes nativos de catalão/espanhol.

Os resultados não revelaram ganhos globais significativos na competência perceptual e produtiva para todos os pares de som examinados, mas indicaram que os informantes do grupo experimental passaram a perceber ou produzir alguns dos sons investigados com mais precisão após o período de instrução fonética. Nesse sentido, percebemos que as sessões de treinamento fonético que os informantes da pesquisa do grupo experimental receberam foram importantes para desenvolver nesses aprendizes uma CF do sistema fonológico da L2 e perceber as diferenças do sistema fonológico de sua L1.

O trabalho de Ruhmke-Ramos (2009) investigou a influência de treinamento e de instrução fonética/fonológica formal na percepção das fricativas interdentais $[\theta]$ e $[ð]$ por brasileiros aprendizes de inglês como L2. Assim como na pesquisa de Mariano (2009), Ruhmke-Ramos (2009) analisou o aprimoramento da pronúncia de L2 com base na relação entre os efeitos da prática de atividades envolvendo as fricativas interdentais [ $\theta$ ] e [ð] na língua inglesa e da instrução explícita a respeito desses sons. Nesse sentido, a instrução explícita atuou de forma a desenvolver a CF dos informantes da pesquisa a respeito dos sons investigados.

Embora os dados tenham apontado que a instrução explícita atuou de forma a desenvolver a CF dos informantes da pesquisa a respeito dos sons investigados, os resultados não foram estatisticamente relevantes para confirmar o efeito positivo do treinamento ou da instrução explícita no desenvolvimento da habilidade de percepção das fricativas interdentais $[\theta]$ e [ð] de língua inglesa por falantes nativos de PB.

A pesquisa de Perozzo (2013) investigou o papel da instrução explícita na percepção de falantes nativos de $\mathrm{PB}$, dos pontos de articulação de oclusivas não vozeadas sem soltura audível [p], [t] e [k], em coda simples finais da língua inglesa. Os resultados dessa investigação mostraram que a instrução explícita mostrou-se significativamente relevante para $[\mathrm{p}]$ e [t] ao comparar o grupo experimental e com o grupo controle da pesquisa, mas não houve dados estatísticos relevantes entre os grupos para confirmar a eficácia da instrução explícita na percepção dos informantes de [t]. Igualmente, o trabalho de Silva (2014a) investigou a percepção de falantes nativos de PB do contraste entre os sons consonantais /s/ - /z/; /1/ - /w/ e /R/ - /r/ do espanhol como L2 e atestou em parte a eficácia da CF nesse processo.

Observamos no Quadro 05, a seguir, um resumo dos trabalhos que investigaram o papel da CF na percepção do sistema fonológico da L2 e, embora os resultados tenham apontado na direção positiva da CF na aquisição fonológica, não foram estatisticamente relevantes.

Quadro 03: Síntese dos trabalhos que investigaram o papel da CF na percepção sonora em L2, selecionados para esta pesquisa, com resultados parcialmente positivos

\begin{tabular}{|lcc|}
\hline \multicolumn{1}{|c|}{ Pesquisa } & L1 & L2 \\
\hline Aliaga-García e Mora (2008) & Catalão/espanhol & Inglês \\
\hline Ruhmke-Ramos (2009) & PB & Inglês \\
\hline Perozzo (2013) & PB & Inglês \\
\hline Silva (2014a) & PB & Espanhol \\
\hline
\end{tabular}

Fonte: Dados da pesquisa (2018).

Na próxima seção, discutiremos a respeito de pesquisas que não confirmaram o papel positivo da CF na aquisição fonológica de L2, com foco na percepção. 


\section{Trabalhos que não confirmam o papel positivo da CF na aquisição fonológica de L2 em termos de percepção}

Em relação aos trabalhos que não observaram um papel positivo da CF na aquisição de L2, podemos mencionar Schwab e Llisterri (2014), que investigaram se um treinamento focado na prosódia do espanhol seria capaz de melhorar a habilidade de falantes francófonos em identificar a sílaba tônica de palavras espanholas. Os resultados mostraram que, embora o desempenho dos informantes francófonos não tenha sido semelhante ao de falantes nativos de espanhol, eles apresentaram a capacidade de incorporar e recuperar, mesmo que de maneira temporária, a acentuação de um pequeno conjunto de pseudopalavras espanholas.

Ao compararmos os resultados do grupo experimental, que recebeu treinamento, com os do grupo controle, sem o treinamento prosódico, observamos, do pré-teste para o pós-teste, uma melhora neste grupo controle, ao passo que não houve melhora no grupo experimental. Podemos compreender esse resultado inesperado, no entanto, pela habilidade dos informantes no treinamento prosódico utilizado.

Observamos no trabalho de Perozzo e Alves (2014) que o nível de proficiência ou o tempo de escolaridade também é visto como um indício de CF, ou seja, aprendizes com proficiência inferior na L2 são caracterizados como portadores de uma menor CF na língua alvo, assim como o inverso é tido como verdadeiro. Nesse sentido, os resultados de Perozzo e Alves (2014) que investigaram a percepção de consoantes sem liberação audível em final de palavra no inglês, por aprendizes brasileiros de inglês como L2, mostraram, no entanto, que o nível de proficiência dos informantes não influenciou na percepção do fenômeno investigado.

Observamos no Quadro 06, a seguir, um resumo dos trabalhos que não observaram um papel positivo da CF na percepção do sistema fonológico da L2:

Quadro 04: Síntese dos trabalhos que não constataram o papel positivo da CF na percepção sonora em L2

\begin{tabular}{|lcc|}
\hline \multicolumn{1}{|c|}{ Pesquisa } & L1 & L2 \\
\hline Perozzo e Alves (2014) & PB & Inglês \\
\hline Schwab e Llisterri (2014) & Francês & Espanhol \\
\hline
\end{tabular}

Fonte: Dados da pesquisa (2018).

A partir desses dados e resultados discutidos nesta seção, compreendemos a importância da CF na percepção do sistema fonológico, durante o processo de aprendizagem de uma L2. Isso se dá, pois, ao perceber os sons da L2 conforme o inventário fonológico da língua alvo, os aprendizes podem compreender que há um distanciamento entre o sistema fonológico de sua L1 e o da língua alvo. Dessa forma, os aprendizes podem desenvolver a habilidade de categorizar cada som conforme o inventário fonológico da língua à qual pertencem.

Assim, essa percepção e compreensão dos sons da língua alvo podem contribuir para que o indivíduo, ao ouvir ou produzir o som adquirido, possa reconhecê-lo ou produzi-lo com base nesse inventário fonológico de L2 e não mais baseado nos sons de sua L1, como, geralmente, acontece nos estágios iniciais da interfonologia dos aprendizes de L2.

Além disso, a produção na L2 pode ocorrer de maneira inadequada por causa de uma não percepção ou não consciência do aprendiz de que determinados segmentos não são produzidos de determinada forma. Diante disso, a instrução com o sentido de desenvolver a CF do aprendiz de L2 das especificidades do sistema fonológico da língua alvo e as diferenças do sistema fonológico da L1 é importante para a aquisição adequada da L2.

Essa consciência do padrão fonológico da L2 permite-lhe diferenciar o sistema fonológico de sua L1 do da língua em processo de aprendizagem. O desenvolvimento dessa CF é possível através de um processo no qual o aprendiz passa a notar ou perceber os sons da língua que está aprendendo. Continuaremos discutindo o papel da CF na aquisição fonológica 
em L2 no próximo tópico, entretanto em uma perspectiva mais da produção ou realização dos sons na interfonologia do aprendiz de L2.

\section{Considerações finais}

Observamos que em $90 \%$ dos trabalhos analisados, a CF teve uma influência na aquisição fonológica de L2. Ainda que, em uma parcela pequena desses trabalhos, os dados não tenham sido estatisticamente significativos para apontar o papel positivo $\mathrm{CF}$ na aquisição da L2, os resultados apontam um direcionamento positivo para a variável em questão.

Conforme podemos observar na maioria dos trabalhos analisados, aprendizes de L2 que apresentam indícios de CF tendem a ter um melhor desempenho na percepção dos sons da língua alvo. Nesse sentido, observamos que a CF habilita os indivíduos a terem uma visão mais ampla do sistema fonológico de sua L1, do sistema fonológico da L2, assim como das semelhanças e diferenças entre esses sistemas fonológicos. Dotados dessa habilidade, os aprendizes têm um desempenho mais eficiente na L2.

Diante disso, parece-nos que fomentar o desenvolvimento da CF nos aprendizes de L2 seja um caminho tão positivo quanto oferecer input em quantidade, qualidade e variabilidade. Como observamos em alguns trabalhos, em determinados momentos, os aprendizes têm acesso a input de qualidade na L2, mas não conseguem fazer as distinções fonológicas necessárias, uma vez que o som é recebido através do crivo fonológico de sua L1. Acreditamos, assim, que o acesso a input amplo e variado, bem como o desenvolvimento da CF na L2 sejam dois fatores complementares e importantes para a aquisição fonológica da língua, uma vez que permitem que essa aquisição seja retroalimentada mutuamente por esses dois processos.

Embora o papel da CF na aquisição fonológica de L2 seja discutível, uma vez que alguns pesquisadores argumentam que é possível a aquisição de modo inconsciente, conforme podemos observar durante a discussão, com base em alguns trabalhos, verificamos que, para a aquisição ocorrer, é necessário algum nível de consciência por parte dos aprendizes. Dessa forma, mesmo que muitos indivíduos não consigam verbalizar nenhum tipo de conhecimento explícito a respeito do sistema fonológico da L2, é possível que momentos de atenção consciente ocorram durante o processo de aquisição para que esta tenha se dado.

\section{Referências}

ALIAGA-GARCÍA, C. AND MORA, J. C. Assessing the effects of phonetic training on L2 sound perception and production. In: RAUBER, A. S., WATKINS, M. A., AND BAPTISTA, B. O. (Eds.). New Sounds 2007: Proceedings of the Fifth International Symposium on the Acquisition of Second Language Speech. Universidade Federal de Santa Catarina: Florianópolis, pp. 10-27, 2008.

ALVES, U. K. Consciência dos aspectos fonéticos/fonológicos da L2. In: LAMPRECHT, Regina Ritter [et al.]. Consciência dos sons da língua: subsídios teóricos e práticos para alfabetizadores, fonoaudiólogos e professores de língua inglesa. Porto Alegre: EDIPUCRS, 2009.

BAKER, W.; TROFIMOVICH, P. Perceptual paths to accurate production of L2 vowels: The role of individual differences. IRAL - International Review of Applied Linguistics in Language Teaching, 44(3), 231-250, 2006.

DARCY, I.; MORA, J. C.; DAIDONE, D. Attention control and inhibition influence phonological development in a second language. Proceedings of the International Symposium on the Acquisition of Second Language Speech. Concordia Working Papers in Applied Linguistics, 2014. p. 115-29. 
GÓMEZ LACABEX, E.; GALLARDO DEL PUERTO, F. Raising perceptual phonemic awareness in the EFL classroom. Proceedings of the International Symposium on the Acquisition of Second Language Speech Concordia Working Papers in Applied Linguistics, 5, 2014. ISSN 2292-4248.

GORDON, J., DARCY, I.; EWERT, D. Pronunciation teaching and learning: Effects of explicit phonetic instruction in the L2 classroom. In J. Levis; K. LeVelle (Eds.). Proceedings of the 4th Pronunciation in Second Language Learning and Teaching Conference 2012. pp. 194-206, 2013.

GUIMARÃES, M. A. Aspectos da fonologia do português como segunda língua por aprendizes anglófonos - uma análise via Teoria da Otimidade. Dissertação (Mestrado em Filologia e Língua Portuguesa), Universidade de São Paulo, São Paulo, 2012.

HUTHAILY, K. Y. Second language instruction with phonological knowledge: Teaching Arabic to speakers of English. University of Montana, 2008. ISBN 0549556389.

KISSLING, E. M. What predicts the effectiveness of foreign-language pronunciation instruction? Investigating the role of perception and other individual differences. Canadian Modern Language Review, 70(4), 532-558, 2014.

KIVISTÖ-DE SOUZA, H. Phonological awareness and pronunciation in a second language. Unpublished doctoral dissertation. University of Barcelona, Barcelona, Spain, 2015.

, H. Brazilian EFL Learners' awareness about L2 Phones: Is Mall Pronounced As 'Mal'?. Trabalhos em Linguística Aplicada, 56(1), 235-258, 2017.

KURT, G.; MEDLIN, J.; TESSAROLO, A. The Perception of Prosodically Ambiguous Intonation Patterns by L2 English Learners and the Effects of Instruction. Proceedings of the International Symposium on the Acquisition of Second Language Speech. Concordia Working Papers in Applied Linguistics, 5, 2014.

LAMPRECHT, R. R.; BLANCO-DUTRA, A.; SCHERER, A.; BARRETO, F.; BRISOLARA, L.; SANTOS, R. M.; ALVES, U. Consciência dos sons da língua: subsídios teóricos e práticos para alfabetizadores, fonoaudiólogos e professores de língua inglesa. 2ed. Porto Alegre: EDIPUCRS, 2012.

MARIANO, M. H. The Influence of Training and Instruction on the Production of Verbs Ending in - ed by Brazilian EFL Learners. Dissertação de Mestrado. Pós-Graduação em Letras Inglês e Literatura Correspondente. Universidade Federal de Santa Catarina: Florianópolis, 2009.

NAVEHEBRAHIM, M. An Investigation on Pronunciation of Language Learners of English in Persian Background: Deviation Forms from the Target Language Norms. Procedia-Social and Behavioral Sciences, v. 69, p. 518-525, 2012. ISSN 1877-0428.

PAGOTO DE SOUSA, M. O. Produção e percepção das vogais e das fricativas $/ \boldsymbol{\theta} / \mathbf{e} / \mathbf{d} / \mathbf{d a}$ língua inglesa por alunos de um curso de Letras. Tese de doutorado. (Doutorado em Linguística e Língua Portuguesa), Universidade Estadual Paulista, Araraquara, 2012.

PELEIAS, F. A. A produção e a percepção da fricativa interdental surda por aprendizes brasileiros de língua inglesa. Dissertação de Mestrado. Mestrado em Linguística Aplicada e Estudos da Linguagem. Pontifícia Universidade Católica de São Paulo - São Paulo, SP, 2009. PEROZZO, R. V. Percepção de oclusivas não vozeadas sem soltura audível em codas finais do inglês (L2) por brasileiros: o papel do contexto fonético-fonológico, da instrução explícita e do nível de proficiência. Dissertação de mestrado. Programa de Pós-Graduação em Letras, Universidade Federal do Rio Grande do Sul, Porto Alegre, 2013.

; ALVES, U. K. Perception of English Word-Final Unreleased Consonants by Brazilian EFL Learners. Proceedings of the International Symposium on the Acquisition of Second Language Speech. Concordia Working Papers in Applied Linguistics, 5, 2014. 
POLIVANOV, E. D. A percepção dos sons de uma língua estrangeira. IN: TOLEDO, D. (Orgs.) Círculo Linguístico de Praga: estruturalismo e semiologia. Porto Alegre, Editora Globo, p. 113-128, [1931] 1978.

ROCCA, P. D. A. A Tecnologia de Fala Aplicada ao Ensino de Entoação da Língua Inglesa para Falantes Nativos de Língua Portuguesa. Tese de doutorado. Lingüística Aplicada e Estudos da Linguagem: Pontifícia Universidade Católica de São Paulo, São Paulo, 2003.

RUHMKE-RAMOS, N. K. The effects of training and instruction on the perception of the english interdental fricatives by Brazilian EFL learners. Dissertação de mestrado. Programa de Pós-Graduação em Letras/Inglês e Literatura Correspondente, Universidade Federal de Santa Catarina, Florianópolis, 2009.

SAMUEL, A. G. KRALJIC, T. Perceptual learning for speech. Attention, Perception \& Psychophysics. 2009, 71 (6), p. 1207 - 1218.

SANDES, E. I. A. Análise das dificuldades dos estudantes brasileiros de E/LE na percepção e na produção dos sons aproximantes e nasais em língua espanhola. Tese de doutorado. Programa de Pós-Graduação em Língua Espanhola e Literaturas Espanhola e Hispano-Americana. Universidade de São Paulo, São Paulo, SP, 2010.

SCHWAB, S.; LLISTERRI, J. Does Training Make French Speakers More Able to Identify Lexical Stress? Proceedings of the International Symposium on the Acquisition of Second Language Speech. Concordia Working Papers in Applied Linguistics, 5, 2014.

SILVA, F. S. D. Consciência fonológica em língua estrangeira: um estudo acerca do processo de aquisição de espanhol por falantes brasileiros. Dissertação de mestrado. Programa de Pós-Graduação em Letras. Universidade Católica de Pelotas. Pelotas, 2014a.

SILVA, S. M. D. Aprendizagem fonológica e alofônica em L2: percepção e produção das vogais médias do português por falantes nativos do espanhol. Tese de doutorado. Programa de Pós-Graduação em Letras. Pontifícia Universidade Católica do Rio Grande do Sul. Porto Alegre, 2014b.

SILVEIRA, R. The influence of pronunciation instruction on the perception and production of English word-final consonants. Tese (doutorado em Letras). Programa de Pós-Graduação em Letras/inglês e literatura correspondente. Universidade Federal de Santa Catarina. Florianópolis, 2004.

SILVEIRA, R.; ALVES, U. K. Noticing e instrução explícita: aprendizagem fonéticofonológica do morfema '-ed'. Nonada, Porto Alegre, v. 13, p. 149-159, 2009.

TRUBETZKOY, N. S. Princípios de Fonologia. Madri. Cincel, [1939] 1973.

VAN DOMMELEN, W. A.; HUSBY, O. The Perception of Norwegian Word Tones by Chinese and German Listeners. IN: RAUBER, A. S., WATKINS, M. A., AND BAPTISTA, B. O. (Eds.). New Sounds 2007: Proceedings of the Fifth International Symposium on the Acquisition of Second Language Speech. Universidade Federal de Santa Catarina: Florianópolis, pp. 165-174, 2008.

WONG, S. W. L.; MOK, P. P. K.; CHUNG, K. K.-H.; LEUNG, V. W. H.; BISHOP, D. V. M.; CHOW, B. W.-Y. Perception of native English reduced forms in Chinese learners: Its role in listening comprehension and its phonological correlates. TESOL Quarterly, v. 51, n. 1, p. 7-31, 2017. ISSN 1545-7249.

Recebido em 28/06/2019

Aceito em 22/08/2019 